\title{
Clinical Audit of Management of Food Poisoning Cases Admitted to Assiut University Pediatric Hospital
}

\author{
MOHAMED M. EL-TALLAWY, M.D.; ALAM EL-DIN M. ABDALLAH, M.D. and \\ TAGHREED M. MOHAMED, M.Sc.
}

The Department of Pediatrics, Faculty of Medicine, Assiut University

\begin{abstract}
Background: Is to assess the clinical management of children with food poisoning admitted to Assiut University Children Hospital.

Aim of the Study: The aim of this clinical audit study is to assess how much the adapted protocols of diagnosis and clinical management of children with food poisoning admitted to Assiut University Children Hospital.

Patients and Methods: The target population of this cross sectional study were all children admitted to Assiut University Children Hospital with food poisoning from June 2016 to June 2017. The study data were collected by reviewing sheets of patients with food poisoning admitted to Assiut University Children Hospital during the study duration.

Results: The study included 40 patients with food poisoning. The average age was $7.26 \pm 4.26$ years and ranged from 1 year to 16 years, 20 cases, $(50 \%)$ were males and 20 cases $(50 \%)$ were females, Data of the study showed that AUCH partially followed the reference standards of the study.
\end{abstract}

Key Words: Food poisoning - Seizures.

\section{Introduction}

FOODBORNE illness (also known as foodborne disease and colloquially referred to as food poisoning). It is any illness resulting from the food spoilage of contaminated food, pathogenic bacteria, viruses, or parasites that contaminate food.

Food borne illness is a serious public health problem, CDC estimate that each year 76 million people get sick, more than 300000 are hospitalized and 5000 die as a result of food borne illness. Primarily the very young, the elderly, and the Immunocompromised are affected.

Correspondence to: Dr. Taghreed M. Mohamed, The Department of Pediatrics, Faculty of Medicine, Assiut University
Recent changes in human demographics and food preferences, changes in food production and distribution systems, microbial adaptation, and lack of support for public health resources and infrastructure have led to the emergence of novel as well as traditional food borne disease. With increasing travel and trade opportunities, it is not surprising that now there is a greater risk of contracting and spreading a food borne illness locally, regionally, and even globally.

Food borne illness are prevalent but the magnitude of illness and associated deaths are not accurately reflected by the data available in both developed and developing countries. To fill current data gap, the world health organization, has taken initiative for estimation of the global burden of food borne illnesses [1]

WHO and the center for disease control and prevention (CDC) report every year large number of people affected by food borne illnesses. Globally, an estimated 2 million people died from diarrheal diseases $70 \%$ of diarrheal diseases are foodborne. According to the estimation by CDC in 2000 , annually 325000 hospitalization and 5200 death in USA [2] .

Food borne illness result from consumption of food containing pathogens such as bacteria, viruses, parasites or the food contaminated by poisonus chemicals or bio toxins [3]. Although the majority of the food borne illness cases are mild and self-limiting, severe cases can occur in high risk groups resulting in high mortality and morbidity in those groups [4]

\section{Abbreviations:}

AUCH: Assiut university children hospital.

CDC : Centre for disease control and prevention.

WHO : World health organisation. 


\section{Patients and Methods}

The study included all children admitted to Assiut University Children Hospital with food poisoning from June 2016 to June 2017. Cases of food poisoning were diagnosed according to clinical suspicion from history, physical examination, and laboratory investigation.

History included, 1-Personal history, age, do the patients consumed uncleaned food outside or inside, do other family members have the same symptoms, lastly do patients live in farm or pet contact.

Examination included: Do patient have disturbed conscious level, drowsiness-anxiety, vital signs abnormalities (RR-HR-BP), diarrhea (which type), dehydration (which degree), abdominal pain, and parethesia-motor weakness.

Lab investigations, InducedCBC, kidney function tests, $\mathrm{Na}-\mathrm{K}-\mathrm{Ca}$, Blood glucose, and Stool analysis.

Regarding the Management, it consested of IV line, Naso gastric tube, Gastric wash, Fluid therapy, antibiotics therapy, Correction of electrolytes, and correction of hypoglycemia.

\section{Results}

Table (1) shows that 40 children with food poisoning were included in this study, there average age was $7.26 \pm 4.26$ years and ranged from 1 year to 16 years, 20 cases, $(50 \%)$ were males and 20 cases $(50 \%)$ were females.

Table (2) demonstrates that personal history was completely taken in $100 \%$, of cases history of consumed uncleaned food $100 \%$ and if family members have the same symptoms $62.5 \%$ or not $37.5 \%$.

Table (3) Explains physical examination showing how many patients have disturbed conscious level $100 \%$, drowsiness (yes $22.5 \%$, no $77.5 \%$ ), abnormalities in vital signs (yes $2.5 \%$, no $97.5 \%$ ), diarrhea (yes $77.5 \%$, no $22.5 \%$ ), dehydration (yes $65 \%$, no $35 \%$ ), and abdominal pain (yes $97.5 \%$, no $2.5 \%)$.

Table (4) Shows abnormalities in examination patients having hypotension $2.5 \%$, patients having bloody diarrhea $15 \%$, watery diarrhea $55 \%$, bloody and watery $7.5 \%$ and patients have dehydration mild $37.5 \%$, and moderate $27.5 \%$.
Table (5) shows investigations which were done to patients as CBC (yes $42.5 \%$, no $57.5 \%$ ), KFT (yes $85 \%$, no $15 \%$ ), Electrolytes (yes $85 \%$, no $15 \%$ ), Blood glucose (yes $27.5 \%$, no $72 \%$ ) and stool analysis (no 100\%).

Table (6) show abnormalities appearing in investigation where microcytic hypochromic anemia in $15 \%$, hypoglycemia in $2.5 \%$ and hyponatremia in $12.5 \%$.

Table (7) illustrates that IV line introduced to all patients $(100 \%)$, Naso gastric tube introduced to cases (yes 30\%) (no 70\%), gastric wash was done to all patients (100\%), $100 \%$ of patients had fluid therapy, about $30 \%$ of patients received antibiotic therapy and $70 \%$ did not receive, $12.5 \%$ of patients had correction of electrolytes, lastly, $100 \%$ of patients had correction of hypoglycemia.

Table (1): Patient demographic characteristics. Total No=50.

\begin{tabular}{ll}
\hline Parameters & No. $(\mathrm{n}=40)$ \\
\hline Age (year): & \\
Mean士SD & $7.26 \pm 4.26$ \\
Range (Min-Max) & $15(1-16)$ \\
Sex: & $\mathrm{N}(\%)$ \\
Male, N (\%) & $20(50.0 \%)$ \\
Female, N (\%) & $20(50.0 \%)$ \\
\hline
\end{tabular}

Table (2): History taken from the studied cases.

\begin{tabular}{lcccc}
\hline & \multicolumn{2}{c}{ Yes } & \multicolumn{2}{c}{ No } \\
\hline & No. & $\%$ & No. & $\%$ \\
\hline $\begin{array}{l}\text { 1- Personal history: } \\
\text { • Name }\end{array}$ & 40 & 100.0 & 0 & 0.0 \\
• Age & 40 & 100.0 & 0 & 0.0 \\
$\begin{array}{l}\text { 2- Do the patients con- } \\
\text { sumed uncleaned }\end{array}$ & 40 & 100.0 & 0 & 0.0 \\
$\begin{array}{l}\text { food: } \\
\quad \text { Outside home }\end{array}$ & 22 & 100.0 & 0 & 0.0 \\
$\quad$ Inside home & 18 & 45.0 & & \\
$\begin{array}{l}\text { 3- Do other family } \\
\text { members have the }\end{array}$ & 25 & 62.5 & 15 & 37.5 \\
same symptoms & & & & \\
$\begin{array}{l}\text { 4- Do patients live in } \\
\text { farm or pet contact }\end{array}$ & 0 & 0.0 & 40 & 100.0 \\
\hline
\end{tabular}

Table (3): Examination of studied patients.

\begin{tabular}{lllll}
\hline & \multicolumn{2}{c}{ Yes } & \multicolumn{2}{c}{ No } \\
Do patient have? & No. & $\%$ & No. & $\%$ \\
\hline 1- Disturbed conscious level & 0 & 0.0 & 40 & 100.0 \\
2- Drowsiness-Anxiety & 9 & 22.5 & 31 & 77.5 \\
3- Vital signs abnormalities & 1 & 2.5 & 39 & 97.5 \\
$\quad$ (RR-HR-BP) & & & & \\
4- Diarrhea & 31 & 77.5 & 9 & 22.5 \\
5- Dehydration & 26 & 65.0 & 14 & 35.0 \\
6- Abdominal pain & 39 & 97.5 & 1 & 2.5 \\
7- Parethesia-motor weakness & 0 & 0.0 & 40 & 100.0 \\
\hline
\end{tabular}


Table (4): Notes on abnormalities detected on examination.

\begin{tabular}{lll}
\hline & No. & $\%$ \\
\hline Vital signs abnormalities (RR-HR-BP): & & \\
Hypotension & 1 & 2.5 \\
Diarrhea (which type): & & \\
Bloody diarrhea & 6 & 15.0 \\
Watery & 22 & 55.0 \\
Watery +blood & 3 & 7.5 \\
Dehydration (which degree): & & \\
Moderate & 11 & 27.5 \\
Mild & 15 & 37.5 \\
\hline
\end{tabular}

Table (5): Investigations done to studied cases.

\begin{tabular}{lccccc}
\hline & \multicolumn{2}{c}{ Yes } & & \multicolumn{2}{c}{ No } \\
\cline { 2 - 3 } \cline { 6 - 6 } & No. & $\%$ & & No. & $\%$ \\
\hline 1- CBC & 17 & 42.5 & & 23 & 57.5 \\
2- Kidny function tests & 34 & 85.0 & & 6 & 15.0 \\
3- Na-K-Ca & 34 & 85.0 & 6 & 15.0 \\
4- Blood glucose & 11 & 27.5 & & 29 & 72.5 \\
5- Stool analysis & & & & 40 & 100.0 \\
\hline
\end{tabular}

Table (6): Notes on results of investigations.

\begin{tabular}{lcc}
\hline & No. & $\%$ \\
\hline CBC & 6 & 15.0 \\
$\begin{array}{l}\text { Microcytic hypochromic } \\
\text { Blood glucose }\end{array}$ & 1 & \\
Hypoglycemia & & 2.5 \\
Na-K-Ca & 5 & 12.5 \\
Hyponatremia & 1 & 2.5 \\
Microcytic Hypochromic & & \\
$\quad$ Anemia, Hyponatremia & & \\
\hline
\end{tabular}

Table (7): Treatment and interventions done to patients.

\begin{tabular}{lcclll}
\hline & \multicolumn{2}{c}{ Yes } & & \multicolumn{2}{c}{ No } \\
\cline { 2 - 3 } \cline { 6 - 7 } & No. & $\%$ & & No. & $\%$ \\
\hline 1- Iv line & 40 & 100.0 & & \\
2- Naso gastric tube & 12 & 30.0 & & 28 & 70.0 \\
3- Gastric wash & 40 & 100.0 & & \\
4- Fluid therapy & 40 & 100.0 & & \\
5- Antibiotic therapy & 12 & 30.0 & & 28 & 70.0 \\
6- Correction of electrolytes & 5 & 12.5 & & 35 & 87.5 \\
7- Correction of hypoglycemia & & & & 40 & 100.0 \\
\hline
\end{tabular}

\section{Discussion}

According to Center for disease control and prevention guidelines management of food poisoning our study included 40 patients admitted in AUH ,20 case male and 20 cases female.

\section{Conclusion:}

Food poisoning is an illness caused by eating foods that have harmful organisms in them. These harmful germs can include bacteria, parasites, and viruses. They are mostly found in raw meat, chicken, fish, and eggs, but they can spread to any type of food. They can also grow on food that is left out on counters or outdoors or is stored too long before eating it. Sometimes food poisoning happens when people don't wash their hands before they touch food.

The aim of this retrospective clinical audit study is to investigate how much the protocol of diagnosis and management of food poisoning is practically implemented in $\mathrm{AUCH}$.

The study included 40 patients with food poisoning. 20 cases were males and 20 were females. Data of the study showed that AUCH partially followed the reference standard of the study.

History should be taken carefully from patients or relatives (in our study obtained from all cases).

- Careful examination should be done (done for all cases).

- CBC should be done according to guidelines (only 17 cases done in our study).

- KFT, Electrolytes also should be done (only 34 case done in our study).

- Stool analysis in our study not done for all cases.

- RBS only measured in 11 case in our study.

- According to guidelines gastrics wash and fluid therapy shoud done (in our study done for all cases).

\section{Recommendations:}

To improve the process of diagnosis, management and therefore the outcome of food poisoning in children attending to AUCH the following recommendations are suggested:

1-Clean: Wash your hands often and always before you touch food. Keep your knives, cutting boards, and counters clean. You can wash them with hot, soapy water, or put items in the dishwasher and use a disinfectant on your counter. Wash fresh fruits and vegetables.

2- Separate: Keep germs from raw meat from getting on fruits, vegetables, and other foods. Put cooked meat on a clean platter, not back on the one that held the raw meat.

3- Cook: Make sure that meat, chicken, fish, and eggs are fully cooked.

4- Chill: Refrigerate leftovers right away. Don't leave cut fruits and vegetables at room temperature for a long time. 
5- When in doubt, throw it out: If you aren't sure if a food is safe, don't eat it.

\section{References}

1- KUCHENMULLER, et al.: Population-Attributable Risk Estimates for Risk Factors Associated with Campylobacter Infection, Australia. Emerging Infectious Diseases, 14 (6): 895-901. doi:10.3201 /eid 1406.071008, 2008.

2- BUZBY J.C. and ROBERTS T.: The Economics of Enteric
Infections: Human Foodborne Disease Costs. Gastroenterology, 136 (6): 1851-1862. doi:10.1053/j. gastro. 2009. 01.074, 2009.

3- World Health Organization (WHO) (2011c). Initiative to estimate the global burden of food borne diseases, Information and publication. Retrieved June, 26, 2011.

4- FLEURY, et al.: A descriptive analysis of hospitalization due to acute gastrointestinal illness in Canada, Canadian Journal of Public Health, 99 (6): 489-93, 2008.

\section{المراجمة الإكلينيكية لعلاج التتسمه الغذائى

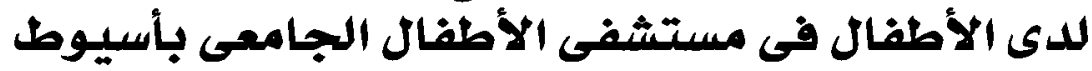

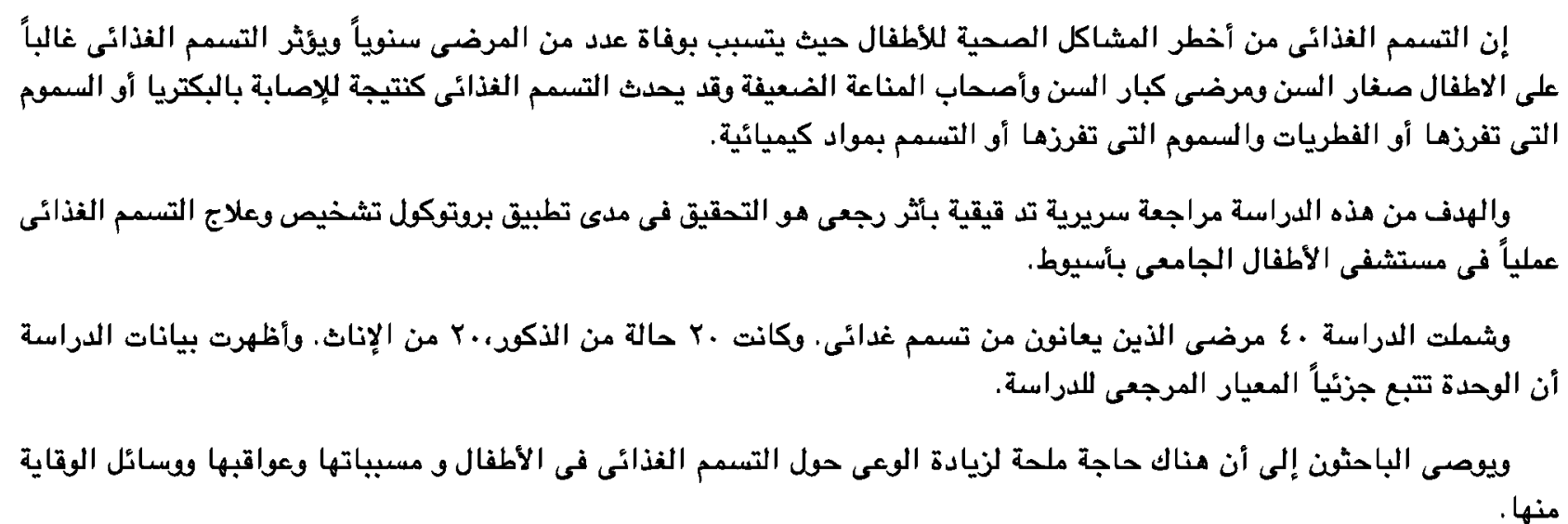

